\title{
Correction to: The knockdown of Neuropeptide FF receptor 2 inhibits capsaicin-induced CGRP Upregulation in mouse trigeminal ganglion
}

\author{
Ya-Tin Lin ${ }^{1,2}$, Zachary Yu ${ }^{3}$, Sze-Chi Tsai ${ }^{4}$, Po-Hung Hsu ${ }^{5,6}$ and Jin-Chung Chen ${ }^{1,2,7^{*}}$
}

\section{Correction to: J Headache Pain 21, 87 (2020) https://doi.org/10.1186/s10194-020-01152-z}

After publication of the original article [1], we were notified of a mistake in the article title. The authors have found that mice knockdown/ or knockout of NPFFR2 showed no responses to capsaicin injection, which upregulated the CGRP protein in the trigeminal ganglion. Therefore, the word "knockdown" should be added into the title to correctly represent the paper's major findings.

The correct title should read:

"The knockdown of Neuropeptide FF receptor 2 inhibits capsaicin-induced CGRP Upregulation in mouse trigeminal ganglion".

\section{Author details}

'Department of Physiology and Pharmacology, Graduate Institute of Biomedical Sciences, Chang Gung University, 259 Wenhua 1st Road, Guishan Dist, Taoyuan City 33302, Taiwan. ${ }^{2}$ Healthy Aging Research Center, Chang Gung University, 259 Wenhua 1st Road, Guishan Dist, Taoyuan City 33302, Taiwan. ${ }^{3}$ Department of Medicine, Chang Gung University, 259 Wenhua 1st Road, Guishan Dist, Taoyuan City 33302, Taiwan. ${ }^{4}$ Department of Biomedical Sciences, Chang Gung University, 259 Wenhua 1st Road, Guishan Dist, Taoyuan City 33302, Taiwan. ${ }^{5}$ Center for Advanced Molecular Imaging and Translation, Chang Gung Memorial Hospital, 5 Fu-Hsing Street, Guishan Dist, Taoyuan City 33302, Taiwan. ${ }^{6}$ Department of Electrical Engineering, Chang Gung University, 259 Wenhua 1st Road, Guishan Dist, Taoyuan City 33302,

The original article can be found online at https://doi.org/10.1186/s10194020-01152-z.

* Correspondence: jinchen@mail.cgu.edu.tw

'Department of Physiology and Pharmacology, Graduate Institute of Biomedical Sciences, Chang Gung University, 259 Wenhua 1st Road, Guishan Dist, Taoyuan City 33302, Taiwan

${ }^{2}$ Healthy Aging Research Center, Chang Gung University, 259 Wenhua 1st Road, Guishan Dist, Taoyuan City 33302, Taiwan

Full list of author information is available at the end of the article
Taiwan. ${ }^{7}$ Neuroscience Research Center, Chang Gung Memorial Hospital, 5 Fu-Hsing Street, Guishan Dist, Taoyuan City 33302, Taiwan.

Published online: 07 December 2020

\section{Reference}

1. Lin YT et al (2020) Neuropeptide FF receptor 2 inhibits capsaicin-induced CGRP Upregulation in mouse trigeminal ganglion. J Headache Pain 21:87. https://doi.org/10.1186/s10194-020-01152-z

(c) The Author(s). 2020 Open Access This article is licensed under a Creative Commons Attribution 4.0 International License, which permits use, sharing, adaptation, distribution and reproduction in any medium or format, as long as you give appropriate credit to the original author(s) and the source, provide a link to the Creative Commons licence, and indicate if changes were made. The images or other third party material in this article are included in the article's Creative Commons licence, unless indicated otherwise in a credit line to the material. If material is not included in the article's Creative Commons licence and your intended use is not permitted by statutory regulation or exceeds the permitted use, you will need to obtain permission directly from the copyright holder. To view a copy of this licence, visit http://creativecommons.org/licenses/by/4.0/. The Creative Commons Public Domain Dedication waiver (http://creativecommons.org/publicdomain/zero/1.0/) applies to the data made available in this article, unless otherwise stated in a credit line to the data. 